\title{
Erratum to: A feasibility study of the use of computed virtual chromoendoscopy for laparoscopic evaluation of peritoneal metastases
}

\author{
Haythem Najah ${ }^{1,2}$ - Réa Lo Dico ${ }^{1,2}$ - Anthony Dohan ${ }^{2,3} \cdot$ Lucy Marry $^{4}$ • \\ Clarisse Eveno $^{1,2} \cdot$ Marc Pocard ${ }^{1,2}$
}

Published online: 12 July 2016

(C) Springer Science+Business Media New York 2016

\section{Erratum to: Surg Endosc}

\section{DOI 10.1007/s00464-016-5028-1}

The metadata for the name of the second author is wrong. The correct given name is Réa and the correct family name is Lo Dico.

The online version of the original article can be found under doi:10.1007/s00464-016-5028-1.

Haythem Najah

haythem.najah@gmail.com

1 Department of Oncologic and Digestive Surgery, Hôpital Lariboisière-AP-HP, 2 rue Ambroise Paré,

75475 Paris Cedex 10, France

2 Sorbonne Paris Cité, CART, INSERM U965, Université Paris Diderot, 74575 Paris, France

3 Department of Abdominal Imaging, Hôpital LariboisièreAPHP, 2 rue Ambroise Paré, 75475 Paris Cedex 10, France

4 Department of Anesthesiology, Hôpital Lariboisière-AP-HP, 2 rue Ambroise Paré, 75475 Paris Cedex 10, France 